\title{
A novel theory of life and its implications on viruses and robots
}

\author{
Yunus A. Çengel* \\ Department of Mechanical Engineering, University of Nevada, Reno, NV, USA
}

\begin{abstract}
A novel theory of life is proposed and its implications on the viruses and the future robots are discussed. The universal laws of physics are inferred phenomena that originate from the observed regularity in the physical realm. An apparent distinct feature of living beings compared to the nonliving ones is the presence of a higher level of regularity, which is indicative of a supplemental set of governing laws within the sphere of life. In this article a living or animate being is defined concisely as a natural entity whose internal changes and external behavior cannot be predicted by the universal laws and forces of physics alone at all times. Everything else is nonliving or inanimate. Likewise, life is defined as a supplemental set of laws and influences that act over a confined space which constitutes the domain of life, superimposed on the universal laws and forces of physics. Also, life is shown to be a field phenomenon like a quantum field, except that life pervades a bounded region rather than the entire spacetime. It is argued that life is an agency with causal power rather than an ordinary emergent property, and that a virus qualifies as a living being. The proposed field theory of life predicts that the future robots are unlikely to acquire life, and that the notion of highly intelligent future robots posing an existential threat to humanity is, in all likelihood, an illusion.
\end{abstract}

Keywords: Life, field theory of life (FTL), laws of life, artificial life, living beings, characteristics of life, animate realm, life of virus, robots with life, nanomachines

\section{INTRODUCTION}

The recent coronavirus pandemic has revived the century-old debate on whether a virus is a living being or not. The reason for this controversy is a lack of clear understanding of the phenomenon of life and its characteristic features. The same reason underlies the heated discussions on the prospect of robots posing an existential threat to humanity. Life is perhaps the most intriguing phenomenon and profound reality in existence. At the same time, it is probably the most evasive occurrence to understand, leaving us all perplexed and puzzled.

It is generally agreed that organisms which possess certain characteristics, such as the abilities to respire, grow, excrete, reproduce, and metabolize, are living beings. But this description is far from being precise since mules do not reproduce, yet only few subscribe to the notion that mules are not alive. The mere existence of controversy over a mule being alive or not is indicative of the dismal state of current understanding of life, and the urgent need for a plausible theory of life.

As a case in point, bacteria satisfy the current criteria of life and pass the test, but viruses stumble. Therefore, while some consider viruses to be alive, most do not. Perhaps the problem lies with the current criteria of life, rather than with the virus, and the plausibility of the current criteria should thus be reassessed critically. Pluto, for example, was recognized as the ninth planet since its discovery in 1930 but was stripped of that title in 2006 when the criteria to qualify as a planet were changed. The same can happen to the virus if we come up with a more fundamental and plausible test of life, one which is clear and easy to apply.

\footnotetext{
*Address. E-mail: yunus.cengel@yahoo.com. 2589-9953 @ 2022 - The authors. Published by IOS Press. This is an Open Access article distributed under the terms of the Creative Commons Attribution-NonCommercial License (CC BY-NC 4.0).
} 
Viruses are often considered to be in the gray area or at the edge of life. Some experts on viruses such as Forterre (2010) insist that a virus is a living organism, while others such as Moreira and López-García (2009) disagree. Most view a virus as a lifeless package of biochemicals or influences rather than living organisms. But the mere existence of the phrase 'biochemical' implies life since a 'biochemical' is just a 'chemical' without life. Precision in language fosters concision and clarity in understanding. The reason viruses are squeezed in a narrow buffer zone that borders chemistry and life is that we do not have the necessary tools to qualify life with precision in a consistent manner. Therefore, opinions abound. As Nobel prize winning cell biologist Paul Nurse (2021) put it, "You could almost say that viruses cycle between being alive, when chemically active and reproducing in host cells, and not being alive, when existing as chemically inert viruses outside a cell. Some biologists conclude that their strict dependence on another living entity means that viruses are not truly alive. But it's important to remember that almost all other forms of life, including ourselves, are also dependent on other living beings."

Life has always been - and it still is - a puzzle that defies decoding and demystification. Not being certain about what constitutes life gives rise to ambiguous phrases such as 'a little alive' and 'borderline life' for viruses. In contrast, there is no such thing as 'a little pregnant' and 'borderline pregnancy' - a woman is either pregnant or not. This is because we know with certainty what constitutes pregnancy. Thus, it is fair to say that the only sure way of ending the ambiguity about distinguishing animate beings clearly from inanimate ones is to correctly answer the fundamental question, 'What exactly constitutes life?'

In the article 'Are Robots Alive?' Cheok and Zhang (2019) expressed the difficulty in their attempt to find a definite answer to this question because of the lack of a suitable definition of life and the many sets of existing criteria of life that are incompatible and even in contradiction with each other: "If we had an authoritative definition of the terms "life" and "alive", and an authoritative list of criteria for life, we would be able to provide an authoritative answer to the question 'Are robots alive?' But as we can see, within traditional biology no such authoritative list or definition exists."

There have been numerous attempts to define life, but they mostly dwell on the physiological processes that occur in living beings. None of the existing definitions of life have found general acceptance as they are perceived as being vague and imprecise. Instead of defining it, life is usually described with a list of features based on biological processes commonly encountered in living beings. As such, life is reduced to chemistry and chemical reactions.

After analyzing 123 definitions of life and searching for commonalities among them, molecular biophysicist E. Trifonov (2011) came up with the following definition: Life is self-reproduction with variations. This definition is also merely an expression of the two of the key features of living beings. It talks about two important functions life performs, but does not say much about life. Despite all the turmoil and confusion about what life is, luckily we all have developed an intuitive understanding of what it is, and we seem to have no difficulty recognizing life when we see it. There is a need to go back to the fundamentals to develop a clear understanding of what life is, and this is what we intend to do in this article.

In a recent comprehensive review article on the major past and current theories of life that include the $(\mathrm{M}, \mathrm{R})$ systems, the hypercycle, the chemoton, autopoiesis and autocatalytic sets, Cornish-Bowden and Cárdenas (2020) have concluded that all of the theories contain some useful features that can be included in a definitive theory of life, but all lack some that are important. As such, they are far from being satisfactory as complete theories of life, and they have generated limited interest. As a result, the search for a more complete theory of life that accurately describes life continues. 
All mainstream descriptions of life are based on the physiological processes observed in living beings rather than underlying fundamental laws of nature. They bear no direct relevance to the main argument in this article, and the interested reader is referred to the above referenced review article for a factual assessment of them. It appears that Erwin Schrödinger, who is one of founders of the quantum mechanics and the recipient of Nobel prize in physics, is the first scientist who saw the relevance of the laws of nature to life based on the observation of the orderly and lawful behavior of matter in living beings, and approached the problem of life from a most fundamental level. Given the success of the laws of physics and the central role they play in physical sciences, he envisioned that perhaps biology also can be fully understood by the discovery of the presently unknown 'other laws of physics' that pervade the animate realm:

"Delbruck's molecular model, in its complete generality, seems to contain no hint as to how the hereditary substance works. Indeed, I do not expect that any detailed information on this question is likely to come from physics in the near future. The advance is proceeding and will, I am sure, continue to do so, from biochemistry under the guidance of physiology and genetics. No detailed information about the functioning of the genetical mechanism can emerge from a description of its structure so general as has been given above. That is obvious. But, strangely enough, there is just one general conclusion to be obtained from it, and that, I confess, was my only motive for writing this book. From Delbruck's general picture of the hereditary substance it emerges that living matter, while not eluding the 'laws of physics' as established up to date, is likely to involve 'other laws of physics' hitherto unknown, which, however, once they have been revealed, will form just as integral a part of this science as the former." (Schrödinger, 1944).

Schrödinger's wish has not materialized as yet. The strength and success of physical science come from its ability to infer and express the regularity in the inanimate realm and the observed natural phenomena as the governing universal laws of physics. Once verified, the complete set of the laws of physics makes it possible to systematically describe all inanimate existence and to predict their future behavior. Without the inferred and expressed laws of physics, the progress in our understanding of the intricacies of the physical realm would be sluggish, and mysteries would abound. Therefore, Schrödinger's insight of 'other laws of physics' for the animate realm is worthy of consideration as the suggested paradigm shift may accelerate progress in life sciences.

In their review paper, Cornish-Bowden and Cárdenas (2020) summarized Schrödinger's inquiry of life as follows: "Schrödinger tried to answer three main questions: 1. How can organisms maintain their organization in the face of a continuous production of entropy as a consequence of the second law of thermodynamics? 2. What is the nature of the hereditary material? 3. Can biology be fully understood (even in principle) in terms of the known laws of physics?"

The authors make the correct assessment that "Schrödinger's third question was the least well understood when he was writing, and remains the least well understood (and the most controversial) today." They add that "The known laws of physics are necessary, therefore, for understanding biological systems, but that does not mean that they are sufficient. The point, strongly emphasized by Rosen (1991, pp. 34-38) and (in conversation) by Varela, is that the universe of biology is vastly larger than the universe of physics. It is perfectly possible that there may be physical laws necessary for understanding biology that cannot be revealed by studying physics alone, because the world that physicists study is too limited."

Walter Elsasser (1961) is another physicist who argued that the laws of physics may not be sufficient for explaining the behavior of living beings. He also viewed the existence of biological laws that cannot be reduced to physics, which he called biotonic laws, as a plausible proposition. Derek Gatherer (2008), who reviewed Elsasser's work, subscribed to the notion of biotonic laws. In the book 'Life 
Itself,' Robert Rosen (1991) pointed out that there is no incompatibility between the laws of physics and Elsasser's biotonic laws: "[Elsasser's] conclusion was that, in a material sense, organisms are governed by their own laws ("biotonic laws"), which do not contradict physical universals but are simply not derivable from them." In this article, we hope to establish that the biotonic laws, which we call the laws of life, underlie the animate realm, just like the laws of physics underlie the inanimate realm.

All the rules and principles associated with natural phenomena are indications of regularity in existence, and are described by the laws of nature, like the laws of physics, as discussed below. Being a natural phenomenon, it is best to describe life as 'laws of life' rather than by another name for consistency since the laws of physics and the laws life together constitute the laws of nature. This also puts life and non-life on comparable platforms.

A concise definition of the phenomenon of life may be hard to come up with, but a factual characterization of life with key traits is achievable by thinking out of the box with a critical eye. A living cell, for example, is more than a lifeless bowl of chemical soup with identical chemical content, including the DNA. That mysterious 'more' which gives the cell its unity, identity, and the ability to perform life functions in a purposive manner is labeled as life. The set of differences that marks a living being just before and just after its death characterizes the life of that being.

After lifetime of research in biochemistry and molecular biology, F. M. Harold (2001) concludes that "The origin of life appears to me as incomprehensible as ever, a matter for wonder but not for explication ... there is much more to this mystery than is dreamt of in molecular philosophy." But he concedes that people are giving up hope for ever unlocking this mystery: "As a subject for serious inquiry, the category 'life' has all but vanished from the scientific literature; it is the particulars of life, not its nature, that fill the numberless pages of scientific journals." Physicist and astrobiologist Paul Davies (2019) concurs: "Even a humble bacterium accomplishes things so amazing, so dazzling, that no human engineer can match it. Life looks like magic, its secrets cloaked by a shroud of impenetrable complexity. Huge advances in biology over the past decades have served only to deepen the mystery."

Many biological scientists equate biology with physical chemistry, and disregard the natural phenomenon of life. Cornish-Bowden and Cárdenas (2020) made the point that "If biologists do not study life, what do they study? As Harold pointed out, biologists study, almost exclusively, details of living organisms, not life itself. The Journal of Biological Chemistry published 20,307 pages in 2018, each of them packed with information, but virtually all of them concerned with small details of living organisms, not with living organisms as such, and none of them asking the question of what life is."

Clearly there is a need to study life from a multidisciplinary perspective without any presupposed notions: "It might be that the complexity of biology will lead to strange and non-intuitive explanations, and to work these out biologists will need ever more assistance from scientists in other disciplines, such as mathematicians, computer scientists and physicists - even philosophers, who are more used to thinking abstractly and are less focused on our everyday experiences of the world." (Nurse, 2021).

This article explores the fundamental phenomenon of life for its own sake and not living beings, and thus the 'unchanging constitution' that govern the animate realm - like the unchanging laws of physics that govern the physical realm since the big bang. Life is posited as a set of 'laws and influences' for the animate realm, in analogy to 'laws and forces of physics' for the inanimate realm. The inanimate existence evolves as evidenced by formation of elements and planets in time and the expansion of the universe, but the laws of physics together with associated forces remain unchanged. The basic physics courses are structured around the understanding of the laws of physics since they are still at work all over the universe, governing natural phenomena in the inanimate realm. The argument here is limited 
to the phenomenon of the unchanging laws of life that govern the animate realm. But first we need to lay the groundwork to ensure clarity in concepts and a common understanding.

\section{REGULARITY IN PHYSICAL REALM AS THE PREMISE TO INFER LAWS OF PHYSICS}

Science is the branch of knowledge which deals with the visible or observable universe that can be perceived by the five senses, and is concerned with the description, prediction, and understanding of the natural and social phenomena on the basis of observations, experimentations, and reason. The two main branches of science are the natural science and social science (sociology, phycology, economics, etc.) Natural science is the branch of science which deals with natural phenomena, and thus it studies the natural world comprised of animate and inanimate existence. The two main branches of natural science are the physical science which studies the inanimate or non-living beings, and life science (also called biological science) which studies the living beings and thus the animate realm. Some add a third category called formal science that is concerned (or just mathematics in the broadest sense) with abstract topics such as mathematics, logic, statistics, and information. But, despite being intertwined with sciences, there is controversy on whether formal science actually constitutes a science since it not based on observations of the tangible physical world and experimentation.

The physical science is comprised of the sub-branches of physics, chemistry, astronomy, earth sciences, etc., each dealing with a different aspect of the inanimate realm, and they are collectively called the physical sciences. Life science is comprised of sub-branches of biology, zoology, botanic, genetics, etc., as depicted in Fig. 1.

The set of rules, principles, and laws associated with the physical sciences such as physics, chemistry, and astronomy and thus govern the inanimate realm (including the dead bodies of living beings) is called the laws of physics. The set of rules, principles, and laws associated with the natural sciences and thus govern all natural phenomena in both animate and inanimate realms is called the laws of

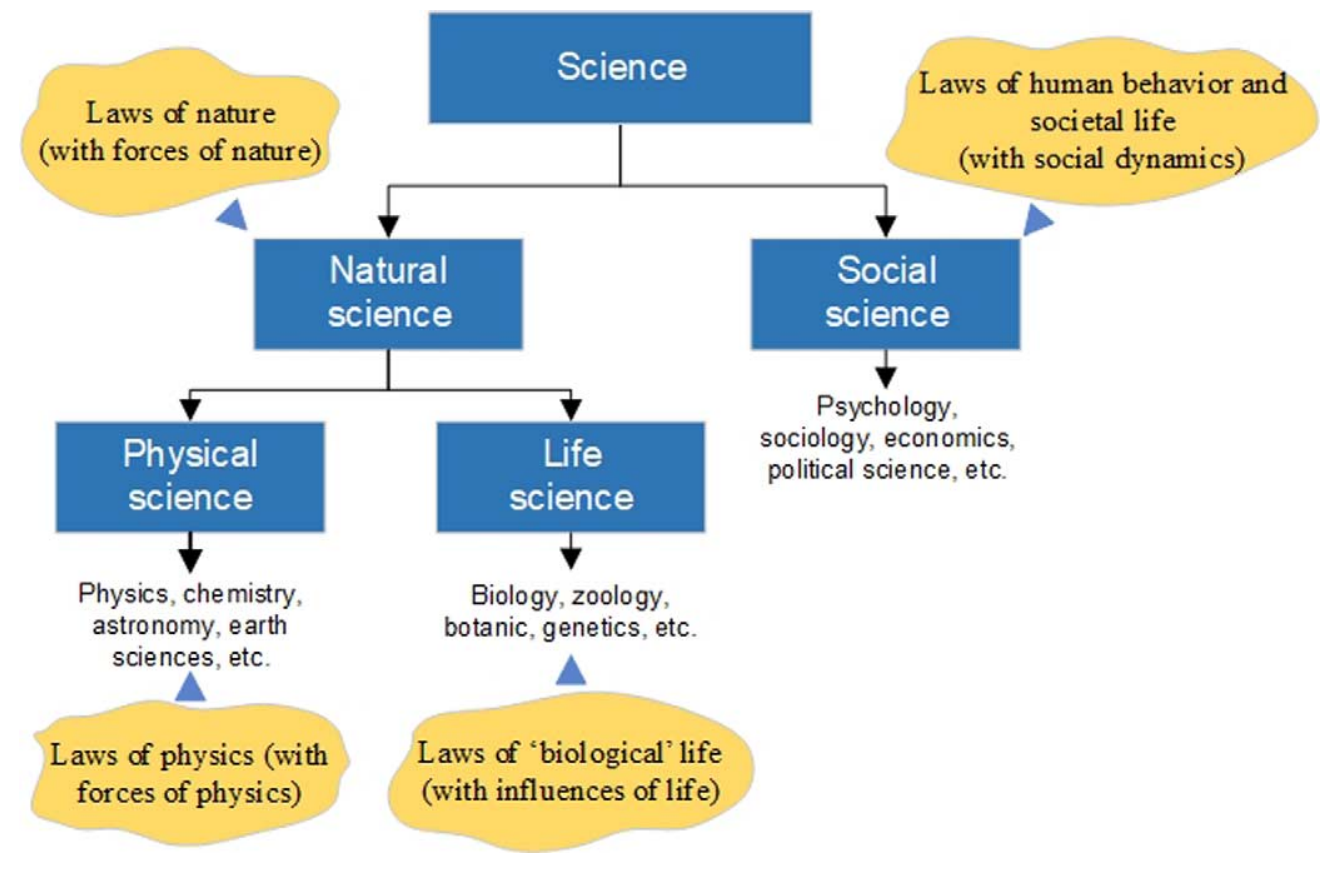

Fig. 1. The branches of science, and the set of governing laws and influences associated with each branch. 
nature. We call the set of supplemental rules, principles, and laws associated with the life sciences and thus govern the animate realm the laws of life. Therefore, the laws of nature are comprised of the laws of physics and the laws of life, just like natural sciences are comprised of the physical sciences and the life sciences.

The universal laws of physics are inferred phenomena that originate from the observed regularity in the physical realm. They are the outcomes of scientific inquiries, and are expressions of order, regularity, and organization in the observable universe. The existence of the laws of physics, which include all the laws, rules, and principles associated with the physical sciences, makes the prediction of future physical events possible. The laws of physics act globally without being selective, and firmly control physical existence via the associated forces of physics. As such, the laws and forces of physics constitute a higher hierarchy in the ladder of existence relative to the physical existence comprised of matter and energy, and they cannot be reduced to matter-energy.

A physical phenomenon is recognized as the effect under the action of an influence exerted on physical existence made of matter and energy, such as water flowing downwards under the influence of gravity, a gas expanding under the influence of heating, and electric current flowing under the influence of higher electric potential. Observed regularity of effects under the action of influences as evidenced by repeatability and predictability are indicators of the presence of underlying laws or principles. As such, the laws of physics are expressions of the regularity of the effects that manifest in the physical realm under the action of influences in a repeatable and predictable manner. The formulations of the laws are simply the cause and effect relations between the causative influences and the manifested effects.

The difference between biology and chemistry is life. If there were no life, there would be no biology or its associated subbranches. Therefore, the existence of life science distinct from the physical science which includes chemistry, is convincing evidence for the existence of life, no matter how elusive and enigmatic life might be. An apparent distinct feature of living beings compared to the nonliving ones is the presence of a higher level of regularity, which is indicative of a higher level of governing laws within the sphere of life. If the rules, principles, and laws of physics were adequate to describe the natural phenomena in living beings, there would be no need to acknowledge life science as a major category of scientific inquiry in addition to physical and social sciences. The current categorization of sciences is evidence that the animate realm involves rules, principles, and laws (or the laws and influences of life for short) that go well beyond chemistry. The phenomenon of death is simply the phenomenon of biology reducing to chemistry. Those biochemicals with their amazing acts reduce to inept chemicals when the elusive life disappears. In fact, this is how we recognize death.

Noting that regularity is a manifestation of underlying laws and influences, the most fundamental feature of life (or biology) must be the laws of life - just like the most fundamental feature of physics is the laws of physics. The former is responsible for the higher-level regularity in the animate realm while the latter is responsible for the base-level regularity in the entire physical realm. A study of cell biology, for example, is essentially a study of the regularity associated with life at the cell level. More specifically, it is the identification and characterization of the laws, rules, principles, influences, and traits of life that manifest in a cell, which are discovered by making logical inferences based on careful observations in the sphere of cell life.

Inanimate beings are fully controlled and governed by the universal laws and forces of physics, such as Newton's laws of motion, the force of gravity, Archimedes' law of buoyancy, Fick's law of diffusion, Ohm's law of electric current, Fourier's law of heat conduction, and Heisenberg's uncertainty principle. Animate beings, on the other hand, appear to be fully controlled and governed by a complementary set of laws and influences of life which act within the domain of the living being, superimposed 
on the universal laws and forces of physics. This is an intuitive and fairly accurate characterization of animate and inanimate realms.

\section{A PROPOSED THEORY OF LIFE}

The universal laws and forces of physics qualify as an agency with causal power since, collectively, they govern the behavior of all physical existence in the entire universe. As Alfred Montapert put it, "Nature's laws are the invisible government of the earth." Likewise, the complementary set of laws and influences associated with life, together with traits that accompany life, can be viewed as the agency of life. The agency of physics is active at every point of spacetime, but the agency of life appears in living beings only and exhibits considerable variation from species to species (Çengel, 2021). After pointing out that living organisms have goals and purposes while atoms and molecules just blindly follow physical laws, Paul Davies attract attention to life acting as an agent: "Life's ability to construct an internal representation of the world and itself - to act as an agent, manipulate its environment and harness energy - reflects its foundation in the rules of logic. It is also the logic of life that permits biology to explore a boundless universe of novelty." (Davies, 2019).

All internal changes and external behaviors of existence and thus all occurrences on the moon, for example, are governed completely by the agency of physics since there appears to be no life there. On earth, however, the occurrences in the inanimate realm are governed by the agency of physics alone, while the occurrences in the animate realm are governed by the agency of life superimposed on the agency of physics. The term 'agency' is used here to characterize the virtual mechanism that appears to be performing certain acts in a repeatable and predictable manner, just like the virtual mechanisms of quantum fields that convert bundles of energy into fundamental particles with a distinct set of properties in a consistent manner (Zee, 2010). Furthermore, particle physicists accurately represent some phenomena observed in the subatomic world such as particle interactions and Casimir forces by 'virtual particles' popping into and out of existence. The quantum field theory gives rise to 'virtual particles' during quantum fluctuations that exhibit some of the characteristics of ordinary particles and appear as internal lines in Feynman diagrams. The transmission of electromagnetic forces, for example, is successfully represented as the exchange of virtual photons in calculations.

In the light of the discussions above, a living or animate being can be defined concisely as follows: A natural entity whose internal changes and external behavior cannot be predicted by the universal laws and forces of physics alone at all times is an animate or living being. Everything else is an inanimate or nonliving being. Likewise, in parallel to the set of laws and forces of physics that act over the entire spacetime, life itself can be defined as a supplemental set of laws and influences that act over a confined space which constitutes the domain of life, superimposed on the universal laws and forces of physics. The domain of life for a living being is the space occupied by the body of that being.

The word 'natural' is used in the definition of living beings to emphasize that: (1) life is a natural phenomenon (it just occurs; it cannot be made - just like the laws and forces of physics are natural and cannot be made) and (2) all manmade technological marvels such as smart devices and softwaredriven micro or macro machinery that mimic living beings are actually nonliving.

It is recognized that the characteristics of living beings can be altered by editing their genomes and even completely replacing them. But this is simply a manipulation of natural life, not the creation of artificial life from nonlife. In the given working definition of life, we assume a state of isolation 
from any willful external interventions and impositions like kicking, charging, driving, and softwarecontrolling so that 'nature' takes its course. The definition further emphasizes that the difference between animate and inanimate beings is life, and thus the set of differences between animate and inanimate beings constitutes the characteristics of life.

These abstract definitions can be visualized by comparing the behavior of a live fish to a dead one in a rough sea. Obviously, the supplemental set of laws and influences discovered by the biological sciences by studying the living beings are at work in the live fish, but they are nonexistent in the dead fish. That is, the internal changes and external behavior of a dead fish in a rough sea can be predicted precisely by the universal laws and forces of physics alone at all times. But this is not the case for a live fish since no mathematical model of physics can predict the behavior of a live fish. Therefore, the live fish and the dead one are like two different interconnected universes with two different sets of laws and forces.

G. K. Chesterton (1925) defined life almost a century ago by contrasting the behaviors of living and nonliving beings and attracting attention to the distinctive feature between them: "A dead thing can go with the stream, but only a living thing can go against it. A dead dog can be lifted on the leaping water with all the swiftness of a living hound, but only a live dog can swim backwards."

It is clear that the universal laws and forces of physics that work perfectly in the inanimate realm as a marvelous predictive tool prove inadequate in the animate realm. This is sufficient observational evidence that life, which is the hallmark of living beings, comes with a localized supplemental set of laws and influences superimposed on the universal laws and forces of physics. And those supplemental laws and influences of life must constitute the distinctive and the most fundamental characteristic of living beings. From this vantage point, biological sciences become inquiries of the supplemental laws and influences of life in the animate realm.

The outcomes of the scientific studies on a particular species, for example, are simply the laws and influences associated with that species. Therefore, the proposed definition of life provides the proper foundational framework for biology and other life sciences. This intuitive characterization draws a clear line between biology and chemistry, and elucidates the vague and blurry notion that life is chemistry, which is the enquiry of inanimate realm at the atomic and molecular level. This way, the biological and physical aspects of the natural phenomena, which characterize the animate and inanimate realms, respectively, complement each other meaningfully and beautifully with clear yet permeable boundaries.

To give an analogy: Living beings resemble autonomous vehicles whose code of operation is severely constrained by the traffic rules and the common driving practices. The driving software of autonomous vehicles with millions of lines of coding constitute their nature, and delineate their code of operation in traffic. The difference between an autonomous vehicle with no human driver and an ordinary vehicle with no human driver on the road is that the former exhibits a will and makes intelligent choices in traffic conditions whereas the latter is governed blindly by the laws and forces of physics with no regard of the traffic laws and rules. The driving software that constrains and controls the motion of a vehicle in traffic represents the purpose of the software engineers that originally wrote the driving software. The autonomous vehicle is governed by a set of superseding laws and rules encoded in the software while the driverless ordinary vehicle is governed only by the laws of physics. In animate realm, life corresponds to the set of superseding laws of life that govern the living beings. The inanimate realm continues to be governed by the laws of physics alone.

It is important to note that the laws of life posited here do not replace the laws of physics; they supplement them. Therefore, the notion of living beings evading the laws of physics and bluntly violating 
them is not correct. After all, all living beings have physical bodies made of matter and exchanging energy, and all matter and energy obey the laws of physics. In living beings, the constituents of the body have an additional set of laws to abide by.

The proposed view of life as a set of supplemental laws and influences superimposed on the laws and forces of physics should remedy misconstrues such as "The law of gravity, for example, is perhaps the most ruthless static pattern of order in the universe. So, correspondingly, there is no single living thing that does not thumb its nose at that law day in and day out. One could almost define life as the organized disobedience of the law of gravity.... If life is to be explained on the basis of physical laws, then the overwhelming evidence that life deliberately works around these laws cannot be ignored.... This would explain why patterns of life do not change solely in accord with causative 'mechanisms' or 'programs' or blind operations of physical laws. They do not just change valuelessly. They change in ways that evade, override and circumvent these laws." (Pirsig, 1991).

There is only one set of universal laws and forces of physics acting over the entire spacetime and thus governing physical existence, but there are as many sets of laws and influences of life as the number of living species, each set acting over the space occupied by all members of a particular species and governing them. Despite the apparent commonalities among all species, each species comes with its own distinct set of laws of life and thus its own constitution. The genome of a species can be viewed as the inscription of the constitution of that species at the cell level. Without the localized laws and influences of life, there would be no science of biology and its subbranches since the universal laws associated with chemistry would be adequate to describe the changes that occur in all beings.

Unity in governance of the physical universe - the universe acting as a cohesive whole in a coordinated manner under one rule - is indicative of a single set of laws of physics acting over the entire universe. Likewise, unity in governance of a living being - the living being acting as a cohesive whole in a coordinated manner under one rule - is indicative of a single set of laws of life acting over the entire domain of living being. The interconnected hierarchical order of governance at the cell, organ, and organism levels - like the hierarchy of governance at the city, state, and country levels - are recognized and distinguished. Life at cell level is the lowest level of life, and it cannot be reduced to the chemical constituents of the cell. Also, life at the organ level is not the combination of the life of the constituent cells since the character and purposive behavior of an organ is very different than that of a cell. That is, there is a top-down order rather than bottom-up. It seems that life at organism, organ, and cell levels are intertwined in a living being and interact in harmony, but life at one level cannot be reduced to the life at the lower level.

Next we present four corollaries.

Corollary 1. From battery-powered toys to robots, smart phones and autonomous vehicles, all manmade devices that are powered by externally-supplied energy such as fuels and electricity are lifeless since the operation of those devices comes to a complete halt when they run out of the externally supplied energy. The behavior of a robot with a completely discharged battery, for example, can be fully predicted by the universal laws and forces of physics. When the battery is recharged and the robot is turned on, the robot will act in accordance with the laws and influences encoded in its software, which constitute the artificial agency of robot. The behavior of living being is also determined solely by the laws and forces of physics when they run out of energy and die. But unlike smart devices that 'die' metaphorically when they run out of energy, a living being can never be brought back to life once it dies, no matter how much energy we supply. As such, the natural agency of life is characteristically different from the artificial agency of robots and other smart devices. When the battery of a robot or a smart device completes its useful life and is no longer rechargeable, it can be replaced by a new 
battery which revives the robot or the smart device. But when life departs from an organism and the organism dies, there is nothing that we can replace in the organism and bring it back to life. Life is not a replaceable energy storage device.

Corollary 2. Dormant entities like plant seeds are categorically alive since they germinate and sprout when planted, and thus undergo internal changes that cannot be foreseen by the universal laws of physics. The changes that a plant seed undergoes during germination are specific to its species.

Corollary 3. Beings whose external behaviors are governed by the universal laws and forces of physics, but not their internal changes - such as people in coma, harvested fruits and vegetables, and even the cuttings of branches - are also alive. This can be verified experimentally - like the cuttings of a rose inserted into soil under proper conditions starting to grow roots. But the hierarchical order of life at cell, organ, and organism levels is recognized.

Corollary 4. Physical systems that are assembled out of nonliving entities - like a storm forming and growing by gathering air and water molecules - are not alive, even if they exhibit chaotic behavior at some point and their behavior appears to be unpredictable before they disperse, or, metaphorically speaking, 'die out.' This is also the case at the subatomic level because of the indeterminacy due to Heisenberg uncertainty principle, which is part of the set of laws, rules, and principles of physics. Our inability to predict the internal changes and external behavior of a chaotic system does not render it a living thing.

Also, chaotic systems involve random disordered fluctuations and lack definite boundaries and purposive or goal-oriented behavior. Afterall, life is ultimate order in internal structure with purpose, and order is the opposite of chaos. Unlike chaotic systems, all living beings resemble highly organized factories operating in perfect order and harmony under one rule and serving a shared purpose.

\section{CHEMICAL REACTIONS AND THE AGENCY OF LIFE}

Life is commonly viewed as a series of chemical reactions since all biological systems are sites of intense chemical activity. But no chemical reaction has ever resulted in any form of life. Two things always appearing together does not necessitate that one be the source or cause of the other. A person blinded by cataract can regain his or her eyesight by transplanting the clouded lenses with clear artificial ones. But this does not mean that the lenses are the source of vision. No transition from chemistry to biology has ever been observed in nature or in lab settings. The common observation that no chemical reaction has ever produced life constitutes an unsurmountable body of evidence that life does not originate from chemical reactions and that life cannot be reduced to chemistry.

In the words of the theoretical physicist $\mathrm{H}$. Haken (2006), "But the more we are dealing with complex systems, the more we realize that reductionism has its own limitations. For example, knowing chemistry does not mean that we understand life." Cornish-Bowden and Cárdenas (2020) agree: "the time has come to move beyond the idea that a system can be fully understood in terms of the properties of its components. Instead we need to understand the functions of the parts in terms of the whole."

Both animate and inanimate beings involve chemical reactions. In animate beings, the chemical processes are tailored towards specific outcomes. The chemical reactions in an animate being resemble the reactions in a well-running chemical factory with a governing agency of factory characterized by unity, order, full coordination, authoritative governance, purpose, knowledge, and ability. Even if 
we don't see the management physically on the production floor, we envisage its presence throughout the factory from the apparent order and the implementation of the rules and regulations, and the orderly and purposeful acts of the workers, machinery, and chemicals. Obviously, the changes that the raw materials undergo in a chemical factory cannot be predicted by the universal laws and forces of physics, since the same raw materials undergo different changes in different factories.

As cell biologist Paul Nurse (2021) explains, "Some of the cell's enzymes that control these reactions work at an astonishingly fast rate, rattling through thousands, even millions, of chemical reactions every second. These enzymes are not only extremely rapid, but can also be extremely precise. They can manipulate individual atoms with a level of accuracy and reliability that chemical engineers can only dream of." He also points out that enzymes work together to ensure that the product of one reaction is passed on directly to become the substrate for the next, resembling assembly lines in a factory, with tiny molecular robots hauling cellular components to where they are needed. Further, the number of different chemical reactions used in a cell is hundreds of times greater than used even the largest industrial chemical plant.

If the piles of raw materials were just mixed and left unattended instead of being routed into the factory, we would still have some chemical reactions occurring in accordance with the universal laws and forces of physics. But those sporadic and purposeless reactions would not constitute a chemical factory. So, what distinguishes a chemical factory from a site where similar chemical reactions occur is the agency of factory composed of a set of laws and influences equipped with the qualities of purpose, knowledge, and skill to convert the selected incoming raw materials to the intended specific products.

From a basic cell to a most complex organism, the agency of life plays the role of authoritative management in a chemical factory. The life of an apple tree, for example, apparently comes equipped with a virtual apple manufacturing facility - selectively taking in ordinary materials from the environment and putting out colorful fruits. The humble apple tree is an indication of the unimaginable technological levels that we can reach by unveiling and manipulating the inferred virtual mechanisms that come hidden with plants or other lifeforms. In the case of an apple tree, which resembles an apple manufacturing facility, the agency of life not only operates this well-run facility, it also constructs it from the scratch one molecule at a time per blueprints of DNA using additive manufacturing. When the enigmatic 'agency' disappears, the entire orderly operation comes to a halt in both a factory and a living being, and the constituents of both entities disintegrate into their physical constituents.

The DNA is simply a large chemical molecule made of atoms, and despite the common thought, it is not alive. The encoded instructions on the DNA are mere symbols of information, and do not qualify as active agents with causal power. Those instructions cannot even know what they are, let alone perform the instructed acts. This is like the instructions on a pizza recipe not being able to get hold of the ingredients and make a pizza. If the ingredients in a kitchen are turned into a cooked pizza, there must have been a real or virtual cook in the kitchen with intent, knowledge, and skill. The universal laws and forces of physics obviously cannot make pizza out of the ingredients and cook it in an oven by following the instruction on the recipe. Likewise, a virtual agency, called the agency of life, must exist in a plant to facilitate the development of the plant precisely as prescribed on the DNA. 


\section{PROPOSITIONS ON THE CHARACTERISTICS OF LIFE}

Next we list the core characteristics of life as six propositions.

First proposition: Life exists. It just does - just like the law and force of gravity exist. Life belongs to the same category of existence as the causal laws of physics. The easily identifiable stark differences between living and nonliving beings are sufficient observational evidence and justification for the plausibility of this proposition. There are no known causal effects of life and thus no known ways to make life out of nonlife. The existence of life is the common thread of all the theories about the origin of life. The notion of the existence of life just recognizes what is, and lays no claims about its origin. Granting life the status of existence ontologically establishes the legitimacy for direct scientific inquiries of life and challenges the notion of life as an illusion. This proposition does not change the fact that the nature of life is still an intriguing mystery. But not knowing the nature of something is not a valid reason for not accepting the existence of that thing.

This is similar to the proposition about the existence of the dark matter and dark energy on the basis of observed influences on known ordinary matter. They are no less elusive than life. Having no idea about the natures of dark matter and dark energy did not keep physicists from recognizing their existence. Enigmatic life deserves the same treatment. Furthermore, we do not have any second thoughts about basing the highly successful quantum field theory on a slew of intertwined and elusive quantum fields, each of which pervades the entire spacetime and acts like a virtual concise particlegenerating mechanism at every point of spacetime. The proposed agency of life fits nicely into this family of elusive fundamental threads out of which the fabric of the realm of existence is interwoven.

Second proposition: Life is a field phenomenon, like quantum fields, except that the domain of influence of the life field is the animate realm, and not the entire spacetime. The contrast between the animate and inanimate realms makes the life field more discernable and with definite boundaries compared to other fields. Note that the observable and well-ordered influences exerted on matter within the domain of life cannot be reduced to the universal laws and forces of physics since those life influences that resemble an invisible mechanism simply disappear when the living organism dies and life disappears. Only life appears to give rise to life, which puts life into a different category of ontology. The field concept borrowed from physics has been an inspiration for other field theories as well (John, 2001).

All observations confirm that life exerts a dominant influence on matter in the sphere of influence of life, similar to a magnet influencing and aligning particles of iron in its domain of influence. For example, a DNA molecule can be built in a lab by assembling the proper molecules. But the synthesized macromolecule just sits there as a pile of atoms, like the molecules in inanimate things, and does nothing. Being a cluster of atoms like other polymers, the DNA is inherently incapable of performing any of the acts inscribed on it. Inserting a synthetic DNA into a manmade cocktail of enzymes, ribosomes, and all other necessary chemicals does not result in any life activity. After all, a completely artificial cell is simply a bag of chemicals, even if it is chemically identical to a live cell. If we artificially reproduce the entire content of a cell (such as a bacterium) atom-by-atom and molecule-by-molecule, including its membrane, and implant the DNA of the live bacterium into this soup, again nothing will happen - the natural DNA will be treated like a stranger in an artificial cell. There will be no life processes in that manmade soup and no replication of the DNA. It will just float around randomly as a bag of inept chemicals.

On the other hand, if we build the DNA of a cell artificially and replace the natural DNA of the cell with its artificial twin, this inept pile of atoms on the manmade DNA will become active and start doing things that no molecule in a chemistry lab can do - such as making a copy of itself. That is, the 
synthetic DNA must be transplanted into a domain of life for it be functional. Therefore, during the genome transplants, life in cells originate from natural cells by tapping into a natural field of life or connecting to the existing network of life. Obviously, the ability to replicate is not an intrinsic quality of a molecule, it is an acquired quality - acquired externally in a compatible domain or field of life.

Things get even more interesting when we make a change on a segment of the artificial DNA that corresponds to a certain feature before inserting it into the live cell like a bacterium: by simply altering the inscription or code of the DNA, the life functions of the bacterium are altered so that the inscribed feature becomes the new apparent feature of the bacterium. This phenomenon gave rise to the field of genetic engineering, with remarkable disruptive inventions like insulin-making bacteria. This means that the agency of life is bestowed with the abilities to decode and read the new inscription, interpret it, and execute and enforce it over the entire body of the bacterium. This observation shows that life is much more than an ordinary emergent quality that pops up on a collection of matter organized in a certain way and passively qualify the assembly of constituents - it is an active agency with causal power.

As J. C. Venter (2012) explains: "Starting with the digital code we synthesized DNA fragments and assembled the genome. We corrected the errors and, in the end, had a 5,386-basepair piece of DNA that we inserted into E. coli... The E. coli recognized the synthetic piece of DNA as normal DNA, and the proteins, being robots, just started reading the synthetic genetic code, because that's what they're programmed to do. They made what the DNA code told them to do, to make the viral proteins. The virus proteins self-assembled and formed a functional virus. The virus showed its gratitude by killing the cells, which is how we effectively get these clear plaques in a lawn of bacterial cells. I call this a situation where the 'software is building its own hardware.' All we did was put a piece of DNA software in the cell, and we got out a protein virus with a DNA core."

Third proposition: Life is an anomaly in the physical realm since its existence cannot be predicted by the laws and forces of physics and the cause-effect relations. In the all-physical universe made of matter-energy that started with the Big Bang and is regulated by the laws of physics, life should have never occurred. No mathematical model, including the standard model in physics, can predict the emergence of life in simulations. Therefore, life is an apparent external imposition onto the physical existence of matter-energy, just like the laws of physics, except that the sphere of influence of life is local whereas the sphere of influence of the laws of physics is universal. Furthermore, life is selective whereas the laws of physics are not. Life, like the laws of physics, does not stem from the material universe, and cannot be reduced to it.

Fourth proposition: The notion of being an animate and inanimate being is a transient or shifting phenomenon. What is an inanimate being today can be part of the body of a living being tomorrow and become animate. Likewise, an animate being can die and become inanimate. This is not surprising since the life feature of animate beings is acquired, not inherent. A particular form of life, bundled with appropriate features, appears on a physical entity which is attuned to that particular form of life out of nowhere, and disappears into nowhere when the physical entity is no longer amenable to life.

When we drink water, which is lifeless, it enters the sphere of influence of life, and becomes subject to the laws and influences of life. As a result, water acquires life and starts behaving like it is 'alive.' A chemical molecule becomes a biomolecule when it joins the body of a living being. But when the same water drips off our face as we sweat, it loses the quality of life, and returns back to being ordinary lifeless matter, as shown in Fig. 2 (Çengel, 2020). These notions are not easy to grasp at first, but this is exactly what is observed in nature. We can predict precisely what water will do in the inanimate realm by the laws of physics alone, but this is not the case in living beings. Another set of 


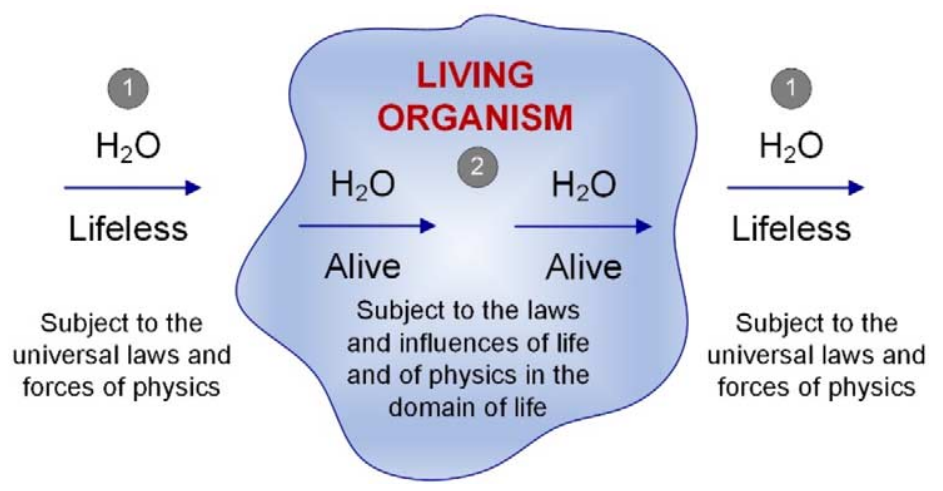

Fig. 2. Matter is lifeless before entering the body of a living being (1), acquires life when in the body (2), and becomes lifeless again when it leaves the body (3).

laws needs to be invoked in living beings, which is labeled as life. This simple observation confirms indubitably that life is best described as an enigmatic field or domain with definite boundaries.

Fifth proposition: Life is an agency with causal power, rather than an emergent property of assemblies of matter that just characterizes the assembly. Life qualifies as an active agent that rules matter and fully governs the material content within its sphere of influence. When two protons are fused together, the combination automatically acquires the properties of helium. When the two protons of helium are separated, each proton acquires the properties of hydrogen. Such properties that emerge out of nowhere passively qualify the assembly of matter. In contrast, an agency actively rules assemblies of matter. An agency has the capacity to subjugate and manipulate physical entities, not just qualify it.

Emergence is described as the phenomenon of a property or quality appearing out of nothing on a physical entity when it is assembled from its parts, and disappearing into nothingness when the entity is disassembled into its parts. Therefore, emergent qualities are the properties of physical beings that just appear out of nowhere, and disappear into nothingness when the physical thing is taken apart. That is, emergent qualities do not originate from the physical constituents of an entity, assembly, or organization.

It is common observation that many properties of the assemblies of matter emerge out of nowhere, since they cannot be reduced to the constituents of the assembly. Most properties of water, for example, cannot be tracked back and related to the properties of hydrogen and oxygen gases. Emergent quantities do not have a stand-alone external existence of their own; their existence depends on the existence of a suitable physical entity to manifest on. Emergent quantities are commonly called emergent qualities since they simply qualify physical entities. They vanish when the physical entity no longer is. The qualities of water, for example, disappear when water is split back into hydrogen and oxygen during electrolysis, and reappear when the process is reversed. The emergent qualities are pinned on the physical things that they appear on in a deterministic fashion, and they qualify the physical entities in a repeatable and predictable manner (Çengel, 2021).

It appears that life perpetuates; it does not emerge. That's why we cannot make life from nonlife. We cannot assemble any material thing which will beget life as a property and become alive. We have to tap into existing enigmatic life to make a living being. For example, it is possible to make something with the properties of water: just assemble and chemically bond hydrogen and oxygen atoms. The properties of water will automatically emerge, and the assembly will acquire the properties of water. But it is impossible to make something with the attribute of life - such as an E.coli bacterium. We can make an exact replica of a bacterium by assembling the material constituents of it atom by 
atom and molecule by molecule, but life will never emerge on that assembly which is materially indistinguishable from a live bacterium. This is sufficient observational evidence that life is not an emergent quantity or a property of organizations.

Philosopher E. F. Schumacher explains the implausibility of the notion of reducing life to an emergent property of assembly of matter with a metaphor as follows: "To say that life is nothing but a property of certain peculiar combination of atoms is like saying that Shakespeare's Hamlet is nothing but a property of a peculiar combination of letters. The truth is that the peculiar combination of letters is nothing but a property of Shakespeare's Hamlet. The French or German versions of the play "own" different combinations of letters." (Schumacher, 1977).

Sixth proposition: The capacity to die is a unique, distinctive, and characteristic feature of living beings. A thing that cannot experience death, cannot be alive. All beings that are bestowed with life are born and eventually die. They have a beginning and an end. Every animate being dies and every being that dies was once an animate being. Nonliving things cannot be killed. Of course, metaphorical use - i.e. 'killing a project' or a cell phone 'being dead' - is excluded. Both seeds and viruses can be killed, and thus they are living beings by this proposition. A virus dies when its protective protein cover is damaged, and repairing the damage or replacing it with a new one does not restore life to a dead virus.

The phenomenon of death is simply the process of permanently reverting back to the inanimate state, and thus being fully controlled by the universal agency of physics. Once biology reduces to chemistry, there is no coming back. It is a one-way street - at least for now. Obviously, the virtual life machinery disappears with death for good. We can predict the motion of a dead fish (or a plastic toy fish) in a rough sea precisely on the basis of the universal laws and forces of physics alone, as stated before; but we cannot do that for a live fish since the actions of a living being are governed predominantly by the laws and influences of life. The only way for chemistry to return to biology is to knock at the door of a compatible lifeform and pledge allegiance to the laws of life that reign there.

It is recognized that a cell may perpetuate through binary fission, but this does not make it immortal. We can take any living monocellular organism and kill it. Once dead, it can no longer be killed, confirming the proposition. The genetic material alone is not alive of its own, as stated before, and cannot be activated unless it enters into a suitable domain of life. This is part of the deep mystery of life that puzzles us all.

\section{DORMANT SEEDS, VIRUSES, AND ROBOTS AS TEST CASES}

A healthy natural seed and an exact artificial replica of it are chemically identical, and thus we may be tempted to say that a seed is not alive. Yet, if we bury both into moist soil, the natural seed germinates and sprouts while the artificial one just sits there, waiting to rot (Fig. 3). Therefore, dormant entities like seeds that show no signs of life are also animate beings since, unlike ordinary inanimate beings, dormant entities obviously come with an imbedded enigmatic agency which will turn them into a fullfledged living being when conditions become conducive to germination and sprouting. Potentially to become plant is indicative of life, and a seed without life cannot do any of that since it is a capsule of lifeless chemicals. Furthermore, the inner structure of seeds changes when the seeds of different plants germinate in ways that cannot be predicted by the universal laws of physics.

If we consider the seed to be nonliving, as some assert, then how can we account for it sprouting and turning into a live plant? Declaring a natural seed to be nonliving is equivalent to declaring it to be a mere chemical compound, which is far from what we observe. An inanimate object cannot 


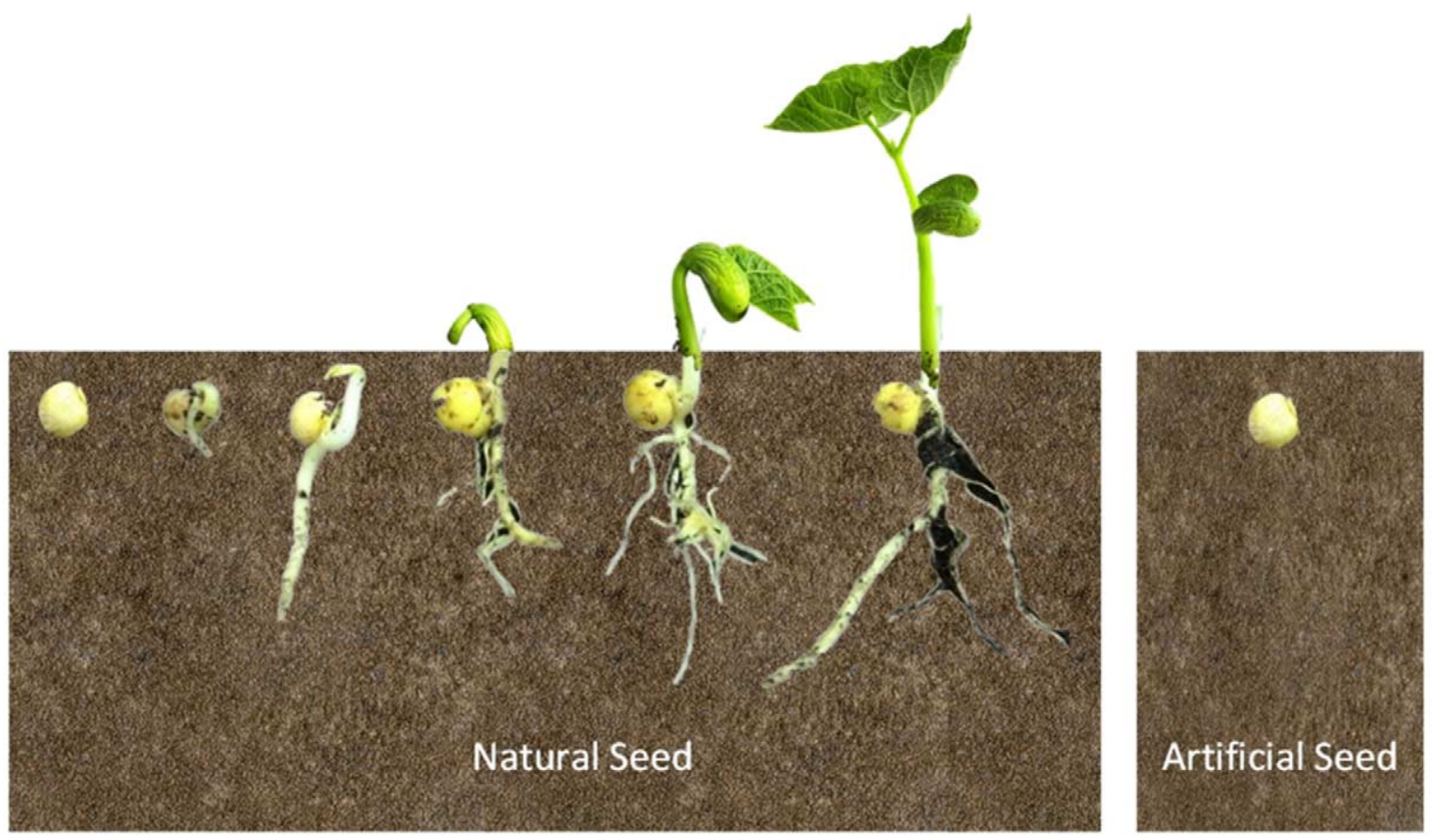

Fig. 3. A natural (live) plant seed with a healthy DNA buried in moist soil germinates and sprouts. But the exact artificial (lifeless) replica of it with the same healthy DNA buried in the same soil does not.

be considered to be animate at the same time. If a seed is inanimate, then it cannot germinate when buried in moist soil; it will disintegrate just like other buried organic materials. The claim that a seed is lifeless is not logically consistent; it does not conform with observations; and it does not comply with our previous experiences. If a seed does not fit into the current depiction of a living being, then the current description of life is inconsistent with reality and needs to be changed.

A similar argument can be given for a virus. A bacterium generates the energy and the biomolecules needed to sustain itself, but a virus cannot. A virus needs to hijack and parasitize a fully functional cell in order to thrive and proliferate. When outside of a host cell, a virus is inactive, just as the seed of a plant is dormant. But when a virus enters a host cell, that sleeping beauty suddenly sheds its coat and bares its genetic material, turning into an active monster. The virus seizes the host cell's machinery and directs it to produce copies of its own genetic material and viral proteins. Then, the produced components are assembled into more viruses, ready to infect other healthy cells. Furthermore, a virus can colonize its host and transpose viral genes to it, which become part of the genome of the species. All these acts characterize a virtual mechanism or machinery that we tag 'agency of life.'

The amount of matter that comprises the physical body of a virus is less than one-billionth of a gram - a miniscule amount by any measure, like a mote of dust. A virus is a collection of atoms and molecules much like any other physical body. It appears to be non-poisonous, and eating a soup made of the atoms and molecules that comprise the virus, including its DNA or RNA, is not likely to cause any harm to the human body, just like eating the sterile dust particles that settle on the food that we eat does not cause any harm. Therefore, a virus is more than a sack of chemicals that include a DNA or RNA molecule, and that a virus qualifies as a lifeform.

Like a seed, a virus behaves as an inanimate object since it is dormant before activation. However, it exhibits the full features of life when it lands on a suitable site where the elusive sleeping agent inside wakes up and all the virtual internal machinery of the virus turn on and power up. Viewing 
a virus as inanimate is equivalent to reducing it to a pack of inept chemical molecules, which are bound to remain as passive entities similar to other chemicals. Lifeless chemicals cannot perform biological functions. Dormant lifeforms and inanimate things cannot be given equal status since the former comes alive under the right conditions whereas the latter remains lifeless under all conditions. This is similar to an unconscious person, who cannot be put in the same category as a dead person, although their appearance suggests otherwise.

To demonstrate that a virus is more than chemistry and thus a living being, let us contrast it with something that is just a chemical and acts as a chemical: the highly toxic chemical compound cyanide. Cyanide chemically bonds with vital molecules in the body that prevent the cells from using oxygen, and thus causes the cells to die out due to the deprivation of oxygen. Oral ingestion of a fraction of gram of solid cyanide or a cyanide solution or exposure to airborne cyanide at a concentration of much less than 1 percent is sufficient to cause death within minutes. Deadly toxic chemicals like cyanide do not multiply and make copies of themselves even under the most favorable conditions. They do not and cannot hijack the machinery of a cell either in order to thrive or proliferate. Unlike viruses, toxic cyanide compounds don't become ineffective when their covers are damaged or removed. To the contrary, cyanide molecules pose a greater threat when they are mixed with air, water, or food.

The constituent chemicals of a virus, including their genetic material, are benign and completely harmless. The typical hamburger that we consume as food, for example, contains millions of DNA and RNA molecules, with no adverse effect except for those who are allergic to meat. The same is the case for pollens that we inhale. Chemically, the constituents of a virus are not much different than the constituents of the cells in our bodies, but obviously the virtual machinery inside a virus is very different. If the virus were just a bunch of chemicals in a protein cover and thus an inanimate entity, it would probably act like a speck of dust and we wouldn't have the word 'virus' in English since it wouldn't be anything with a distinctive feature. What makes a virus what it is, is the invisible agency or the virtual mechanism that is characteristic of life.

To make the case of a virus being alive more strongly, let us carefully tear the cover of a virus and pour its entire content, including its DNA or RNA molecule on a piece of paper - a process that is equivalent to killing the virus and turning it into a pile of disjointed chemicals. Now, let us inhale the entire contents of the virus, including the DNA or RNA, so that the cells in the linings of our lungs are exposed to the genetic material of the virus. If our arguments that a virus is more than chemistry is true, our lungs will treat all the constituent molecules of the virus as dust particles and just discard them. Or, to make things simple, if we extract the genetic material of viruses, prepare an aerosol rich in DNA or RNA molecules, and then inhale it, again those marvelous macromolecules will be treated as specks of inept matter, similar to dust particles, and nothing will happen.

It is recognized that the genome of a virus will come alive when it is injected into an appropriate cell. But this requires an intentional and concerted effort. Genomes are inherently lifeless chemicals, and if thrown into an organ like a lung or a heart, no genome will attempt to pierce the membrane of a cell, enter it, and annex the cell. In fact, the recently developed mRNA-based vaccines to fight Covid-19 do just that: a fragment of the virus's genetic material is injected into the body, tricking the body into thinking that the virus is there and it is being attacked by the virus, and it mounts an immune response with antibodies by producing the protein which is present on the surface of the coronavirus. This new vaccine turned out to be safer and more effective than the traditional vaccines which involve the injection of a piece of a virus, a dead virus, or a weakened virus.

All constituents of a living being, including the genome, are inherently lifeless when extracted out of the living being and are isolated. When a virus is disassembled into its constituents, for example, the elusive life disappears, never to return. When the constituents are reassembled with precision so 
that the assembly perfectly resembles the original virus, life never reappears and the assembled virus never exhibits purposeful behavior. It just stands there as a pile of physical components. Therefore, the notion that the assembled constituents suddenly acquire life out of nowhere is far from being factual. The common observation that life emerges only from life, and never from nonlife, provides convincing evidence for the existence of enigmatic life.

When we inhale viruses and allow them to enter our body intact, those inactive little creatures wake up and start doing their thing within the cells that they occupy and thrive on, acting as active agents in accordance with their own set of laws and influences, exhibiting all signs of life as we know it. Noting that the physical realm consists of animate and inanimate realms, and contrasting the virus with cyanide, we can observe that the virus clearly does not belong to the inanimate world. Observations confirm that viruses are governed and characterized by the laws and influences of life.

Finally, we all are familiar with the idea of 'fighting' cancerous cells, mosquitoes, bacteria, and viruses. But, excluding the metaphorical use of the term, there is no such thing as 'fighting' chemicals. This is because poor chemicals - even the toxic and explosive ones - just sit quietly wherever they are put, with no ambition to inflict harm. It is absurd to fight chemicals in order to reduce them to chemicals, which would be the case if a virus was considered to be a nonliving entity. The mere act of fighting the virus is an affirmation that the virus is equipped with an active agent, which is indicative of life, and that a virus is not just a bunch of chemical molecules bundled together. When we fight against 'something,' we fight against an active agent with the objective of deactivating it, that is, driving out the 'active agency' which is life. The notion of fighting an army of nonliving viruses is no different than the notion of fighting an army of nonliving soldiers.

It should be pointed out that the lifeless robot armies that we see in the futuristic movies are just the subjects of their human masters. Such armies reflect the purpose, knowledge, and power of their live masters. The robots are like the extensions of a living being with intent. When we fight an army of robots, we are actually fighting the mastermind that directs that army of dummies. If the mastermind is killed, the attack of the robot army comes to an automatic halt. The same argument applies to the armies of drones. Shooting the attacking drones is really fighting the will of the living being that deploys and controls the drones. Once the living beings in the command center are exterminated, the drones will just ground themselves and wait for the instructions of their new master.

\section{VIRUSES VS. NANOMACHINES: THE DIFFERENCE THAT LIFE MAKES}

The simplicity of viruses relative to cells and their amenability to reductionist and mechanist explanations gave rise to the nanomachine metaphor in virology. Viruses resemble tiny machinery since they are purposive and goal-oriented, and are often described as molecular nanomachines. They are sometimes characterized as 'incredible nanomachines' and 'elegantly programmed nanomachines' (Hemminga et al., 2010; Johnson, 2010). There are also those who think the machine metaphor does not do justice to living things, and a more holistic view is needed. Microbiologist C. W. Woese (2004) puts it as "Let's stop looking at the organism purely as a molecular machine. The machine metaphor certainly provides insights, but these come at the price of overlooking much of what biology is. Machines are not made of parts that continually turn over, renew. The organism is. Machines are stable and accurate because they are designed and built to be so. The stability of an organism lies in resilience, the homeostatic capacity to reestablish itself."

But despite the apparent machine-like aspects, viruses are inherently different from nanomachines: the former are alive as argued here, the latter are not. Viruses are natural beings (no human intervention), whereas nanomachines are manmade artificial entities. As such, viruses existed long before the 
humans inhabited the earth, and they will probably continue to do so long after the human race goes extinct. The existence and sustenance of nanomachines, on the other hand, depend on the existence of humans - intelligent and conscious living beings with intent, knowledge, and skill. Associated with every lifeless nanomachine is a living and intelligent 'remote agent' which equips the nanomachine with purpose, information, and ability. If a nanomachine inflicts any damage, its operating agent will be held responsible, not the nanomachine itself. Nanomachines, therefore, are the makings of the living beings, and as such, are the extensions of the sphere of influence of life and the exhibitions of living beings.

There can be no purposive equipment such as the sophisticated nanomachines without human involvement. In fact, even a simple pot or plate wouldn't exist in the inanimate realm without human involvement, as evidenced by archeologists labeling all such unearthed findings during excavations as manmade artifacts rather than natural formations, and displaying them in museums. That is, without life, the earth's surface would be just like the lifeless moon's surface, exhibiting only the makings of the purposeless acts of nature in the physical inanimate realm. This is all that the 'agency of physics' with all the universal laws and forces of physics in its arsenal can do.

If an excavation project is initiated on the moon, no one would expect any pots or pans to be found (unless the astronauts of Apollo 11 dropped them there), let alone highly technological nanomachines (or robots, smart phones, etc.) with sophisticated software. The enigmatic agency which is responsible for the existence of the 'animate realm' with millions of intriguing goal-oriented and sophisticated species is, undoubtedly, what we call 'life.' There is no other plausible candidate to account for the stark difference between the animate and inanimate realms. Therefore, despite the mystery that surrounds it, it is imperative to recognize life as an agency with a set of laws and influences superimposed on the laws and forces of physics.

If something cannot be the making of the acts of nature in the physical inanimate world, that something must be a living being (such as a virus) or the making of a living being (such as a nanomachine or a robot). This should suffice as a plausible argument for the case that a virus with all its sophisticated machinery and secretive coding must be classified as a living being since it cannot be a natural formation of the inanimate realm.

The wavy formations of the silicon-rich sand in a desert is obviously the making of the agency of physics - a natural outcome of the purposeless actions of the laws and forces of physics such as winds. But a purposive silicon chip with millions of interconnected circuits and input/output junctions is not - it requires the imposition of a semiconductor manufacturing facility with its own processing laws, procedures, and effects as an add-on to the laws and forces of physics.

Despite all their sophisticated software and technology that they possess, the operations of all nanomachines would come a complete halt if the human connection is cut off. A stretch film that wraps together all the physical components of a nanomachine, including the battery, is not a nanomachine. Without its software, a fully assembled nanomachine is simply an assembly of parts. A nanomachine with its software loaded and the power switch turned on is still an inactive entity unless a 'go' command is given by a purposive agent externally.

A superficial look may not be able to tell apart a sophisticated life-like humanoid like Erica from a living human being since the humanoid can perfectly mimic a human being with its look, facial expressions, smooth acts, and carrying out an intelligent conversation. But, impressed by the striking resemblance, no one would claim that humans are actually humanoids on the premise that they look alike and act alike. Such a claim equates the denial of the existence of the enigmatic life, and thus the animate realm, and reducing existence to piles of matter and energy governed by the laws and 
forces of physics alone. That claim also equates the diverse existence on the surface of the earth to the existence on the surface of a planet with similar physical conditions to the earth, but no life.

This assessment rebuts the popular notion that someday human-made nanomachines themselves will be self-sustaining and self-replicating, and thus avoiding the need for any human intervention, which is the core concept of the nanomachine metaphor for viruses. As living intelligent beings, we have the power to make machines that make machines, but no machine has the power to reproduce. If a machine is placed into a resource-rich environment, it will not rebuild replicas of itself by consuming materials and energy, and bestow its offspring the ability to reproduce. Even a computer code cannot duplicate or modify itself unless the external coder instructs the code to do so.

We underline that, in the end, without the laws and influences of life that prevail in animate realm, all that the mere laws and forces of physics can do in the inanimate realm are the purposeless accumulations and rearrangements of matter with arbitrary shapes, like the hills and valleys of sand in Death Valley. All observed goal-oriented acts and artifacts, whether natural or human-made, are associated with life.

Finally, bacteria closely resemble nanomachines since they are also molecular machinery that perform specific tasks, like viruses. As such, it is difficult to justify the notion that bacteria are alive but viruses are not - just like it is difficult to justify the notion that donkeys are alive but the mules are not simply because mules do not reproduce.

\section{CLOSING REMARKS}

The reasoned arguments presented in this article on the basis of careful observations of living beings and the inference that life is a complementary set of laws and influences acting in the domain of life and superimposed on the laws and forces of physics constitute a new theory of life. Inspired by the highly successful quantum field theory which is based on a slew of quantum fields, each of which pervading the entire spacetime and acting like a virtual particle-generating mechanism at every point of spacetime, the theory articulated in this article can be called the field theory of life. It qualifies as a viable scientific theory with plenty of conforming observational evidence since its propositions, such as virus being more than chemistry and thus alive, are testable and falsifiable. It will remain a viable theory until it is falsified by making artificial animate beings out of inanimate matter, which is equivalent to creating new artificial laws and influences of nature that act over a confined space and fully control matter within that bounded space of influence. The proposed theory provides fresh new insights into life, and has the potential to bring about a paradigm shift in our understanding of life and the way we view living beings.

The hallmark of a successful scientific theory is its ability to make predictions on top of explaining the observed natural phenomena. Based on the propositions that (1) life is a field phenomenon and comes with a set of laws, influences, and qualities that vary considerably from species to species and (2) certain qualities like consciousness, emotions, and desires are observed only in living beings and never in nonliving entities, we can predict that no machine will ever gain consciousness, emotions, etc. unless they acquire life first. Considering that the essence of life is a set of laws and influences that act within the domain of life, the likelihood of artificially creating life from scratch is no higher than artificially creating a new law of physics and the influence that accompanies it, which can be said to be zero. That is, creating new laws and influences of nature (or changing the existing ones) is beyond human reach - they exist as a priori. Therefore, the field theory of life (FTL) postulates that making artificial life and building living machines appear to be an impossibility. 
The law of conservation of mass and energy, for example, can only be changed by creating mass and energy out of nothing. Also, according to Newton's law of universal gravitation, the force of gravity $F$ between two bodies of mass $m$ and $M$, respectively, separated by a distance $r$ is expressed as $F=G m M / r^{2}$ where $G$ is the gravitational constant. The likelihood of creating artificial life by changing or cancelling the laws and influences of life is no higher than the likelihood of changing or cancelling the law and force of gravity, rendering objects weightless. Philosophically speaking, changing the constitution of a country (and the mechanism to enforce it) that regulates the acts of its constituents of people is a hard problem; but changing the constitution (and forces) of the universe that regulates the acts of its constituents of matter and energy is an impossible problem.

As the Austrian physicist Ludwig Boltzmann said, "Nothing is more practical than a good theory." Considering that traits such as consciousness, intent, emotions, and thoughts appear on living beings only, the proposed field theory of life predicts that regardless of how sophisticated its microprocessor might be, no smart machine will ever gain consciousness and pose an existential threat to humanity since it is beyond human capability to make life artificially. (Besides, artificial intelligence AI is essentially software, which is merely a set of inscribed instructions and commands.) The complete failure of all ambitious projects to create artificial life from nonlife in the first decade of this century supports this proposition. Therefore, assuming this theory passes the test of time, the only way to build living machines is to start with a living species as the platform to tap into life, and to manipulate the species and equip it with desirable traits by implanting artificially made gadgets congruent with life.

The proposed field theory of life can also provide a basis to help resolve the question of when new life begins in humans and other mammals: a fetus can be regarded as a distinct living entity when the life processes in its body are governed by a separate and distinct set of laws and influences than those of the natural mother's body. This is assessed by the ability of a fetus to grow into a fully developed baby outside the natural or surrogate mother's womb. Therefore, one can conjecture that new life begins and a fetus can be regarded as a distinct living being when it can survive outside the mother's womb. Otherwise, the fetus is just a growth of the mother's body as a cluster of live cells and tissues, and not a distinct living entity.

The current state of inquiries in life sciences reminds us of the development period of the steam engines in the $18^{\text {th }}$ and $19^{\text {th }}$ centuries that powered the industrial revolution: an ingenious and highly successful enterprise, but without an underlying fundamental theory for the phenomenon of the conversion of heat energy into motive power or work. Today we owe the high efficiency of the steam engines and the development of other heat engines such as those that power our cars, power plants, and ships to the discovery and formulation of the laws of physics related to the character, conservation, and conversion of energy mostly in the second half of the 19th century. French engineer and scientist Sadi Carnot, one of the founders of thermodynamics, expressed this in 'Réflexions sur la Puissance Motrice du Feu' published in 1824, as follows:

"Notwithstanding the work of all kinds done by steam-engines, notwithstanding the satisfactory condition to which they have been brought today, their theory is very little understood, and the attempts to improve them are still directed almost by chance.... In order to consider in the most general way the principle of the production of motion by heat, it must be considered independently of any mechanism or any particular agent. It is necessary to establish principles applicable not only to steam engines but to all imaginable heat-engines, whatever the working substance and whatever the method by which it is operated.

Machines which do not receive their motion from heat, those which have for a motor the force of men or of animals, a waterfall, an air-current, etc., can be studied even to their smallest details 
by the mechanical theory. All cases are foreseen, all imaginable movements are referred to these general principles, firmly established, and applicable under all circumstances. This is the character of a complete theory. A similar theory is evidently needed for heat-engines. We shall have it only when the laws of Physics shall be extended enough, generalized enough, to make known beforehand all the effects of heat acting in a determined manner on any body." (Carnot; trans. Thurston, 1897).

In recognition of the deep insight of Edwin Schrödinger and in honor of his memory, we conclude this article with an inspirational passage from the last chapter of his 1944 book 'What is Life?': "What I wish to make clear in this last chapter is, in short, that from all we have learnt about the structure of living matter, we must be prepared to find it working in a manner that cannot be reduced to the ordinary laws of physics. And that not on the ground that there is any 'new force' or what not, directing the behaviour of the single atoms within a living organism, but because the construction is different from anything we have yet tested in the physical laboratory.

To put it crudely, an engineer, familiar with heat engines only, will, after inspecting the construction of an electric motor, be prepared to find it working along principles which he does not yet understand. He finds the copper familiar to him in kettles used here in the form of long, wires wound in coils; the iron familiar to him in levers and bars and steam cylinders here filling the interior of those coils of copper wire. He will be convinced that it is the same copper and the same iron, subject to the same laws of Nature, and he is right in that. The difference in construction is enough to prepare him for an entirely different way of functioning. He will not suspect that an electric motor is driven by a ghost because it is set spinning by the turn of a switch, without boiler and steam. If a man never contradicts himself, the reason must be that he virtually never says anything at all." (Schrödinger, 1944).

\section{FUNDING}

This research did not receive any specific grant from funding agencies in the public, commercial, or not-for-profit sectors.

\section{CONFLICT OF INTEREST}

The authors have no conflicts of interest to declare that are relevant to the content of this article. The authors have no relevant financial or non-financial interests to disclose.

\section{REFERENCES}

Carnot, S. (1897). Reflections on the Motive Power of Heat; Thurston, R.H., Translator from French (p. 1897). London, UK: Chapman \& Hall, Ltd.

Çengel, Y.A. (2020). On the ontology of life and mind: A proposed paradigm shift. Int. J. Research, Innovation and Commercialization, 3(1), 47-72. doi:10.1504/IJRIC.2020.109387.

Çengel, Y.A. (2021). On emergent quantities, mental perceptions and constructs, and agencies: A holistic view of existence. J Neurobehav, Sci., 8(2), 157-170. doi:10.4103/jnbs.jnbs_33_21.

Cheok, A.D. \& Zhang, E.Y. (2019). Are robots alive? In Human-Robot Intimate Relationships. Human-Computer Interaction Series, Cham: Springer. doi:10.1007/978-3-319-94730-3_8.

Chesterton, G.K. (1925). The Everlasting Man. New York: Dodd, Mead \& Co. 
Cornish-Bowden, A. \& Cárdenas, M.L. (2020). Contrasting theories of life: Historical context, current theories. In Search of an Ideal Theory. BioSystems 188. doi:10.1016/j.biosystems.2019.104063.

Davies, P. (2019). The Demon in the Machine: How Hidden Webs of Information Are Solving the Mystery of Life. Chicago: University of Chicago Press.

Elsasser, W.M. (1961). Quanta and the concept of organismic law. J. Theor. Biol., 1(1), 27-58. doi:10. 1016/0022-5193(61)90025-X.

Forterre, P. (2010). Defining life: The virus viewpoint. Orig. Life Evol. Biosph., 40(2), 151-160. doi:10.1007/s11084-010-9194-1.

Gatherer, D. (2008). Finite universe of discourse: The systems biology of Walter Elsasser (1904-1991). Open Biol. J., 1, 9-20. doi:10.2174/1874196700801010009.

Haken, H. (2006). Information and Self-Organization: A Macroscopic Approach to Complex Systems (3rd ed.). Heidelberg: Springer.

Harold, F.M. (2001). The Way of the Cell: Molecules, Organisms, and the Order of Life. New York: Oxford University Press.

Hemminga, M.A., Vos, W.L., Nazarov, P.V., Koehorst, R.B., Wolfs, C.J., Spruijt, R.B. \& Stopar, D. (2010). Viruses: Incredible nanomachines. New advances with filamentous phages. Eur Biophys J., 39(4), 541-550. Epub 2009 Aug 13. PMID: 19680644; PMCID, PMC2841255. doi:10.1007/s00249009-0523-0.

John, E.R. (2001). A field theory of consciousness. Consciousness and Cognition, 10(2), 184-213. https://www.ncbi.nlm.nih.gov/pubmed/11414714. doi:10.1006/ccog.2001.0508.

Johnson, J.E. (2010). Virus particle maturation: Insights into elegantly programmed nanomachines. Curr Opin Struct Biol., 20(2), 210-216. Epub 2010 Feb 9. PMID: 20149636; PMCID, PMC2854226. doi:10.1016/j.sbi.2010.01.004.

Moreira, D. \& López-García, P. (2009). Ten reasons to exclude viruses from the tree of life. Nat. Rev. Microbiol., 7, 306-311. doi:10.1038/nrmicro2108.

Nurse, P. (2021). What Is Life? New York: W. W. Norton and Company, Inc.

Pirsig, R.M. (1991). Lila - an Inquiry into Morals. New York: Bantam Books.

Rosen, R. (1991). Life Itself: A Comprehensive Inquiry into the Nature, Origin, and Fabrication of Life. New York: Columbia University Press.

Schrödinger, E. (1944). What Is Life? Cambridge: The University Press.

Schumacher, E.M. (1977). A Guide for the Perplexed. New York: Harper \& Row.

Trifonov, E.N. (2011). Vocabulary of definitions of life suggests a definition. Journal of Biomolecular Structure and Dynamics, 29(2), 259-266. doi:10.1080/073911011010524992.

Venter, J.C. (2012). https://www.edge.org/conversation/j_craig_venter-what-is-life-a-21st-centuryperspective. Accessed July 7, 2017.

Woese, C.R. (2004). A new biology for a new century. Microbiology and Molecular Biology Reviews, 68(2), 173-186. doi:10.1128/MMBR.68.2.173-186.2004.

Zee, A. (2010). Quantum Field Theory in a Nutshell (2nd ed.). Princeton, NJ: Princeton University Press. 